\title{
PAPER \\ Physical Architecture and Model-Based Evaluation of Electric Power System with Multiple Homes*
}

\author{
Yoshihiko SUSUKI $^{\dagger \mathrm{a})}$, Member, Ryoya KAZAOKA $^{\dagger \mathrm{b})}$, Nonmember, and Takashi HIKIHARA ${ }^{\dagger c)}$, Fellow
}

\begin{abstract}
SUMMARY This paper proposes the physical architecture of an electric power system with multiple homes. The notion of home is a unit of small-scale power system that includes local energy source, energy storage, load, power conversion circuits, and control systems. An entire power system consists of multiple homes that are interconnected via a distribution network and that are connected to the commercial power grid. The interconnection is autonomously achieved with a recently developed technology of grid-connected inverters. A mathematical model of slow dynamics of the power system is also developed in this paper. The developed model enables the evaluation of steady and transient characteristics of power systems. key words: power system, architecture, model, bifurcation, uncertainty, system-level design
\end{abstract}

\section{Introduction}

Electricity distribution has been traditionally managed and regulated by regional suppliers. Because the complex physics of electricity distribution occurs on a wide range of scales in both space and time, this is a very challenging task, especially on the demand side. Many researchers have made intensive efforts to overcome this challenge through the integration of information, communications, power, and control technologies. The integration begins at the smallest grid inside a home, then moves to building units, and finally reaches a local community. There are many ongoing research projects on electricity distribution such as the socalled Smart Grid vision: see e.g. [1]. Thus, it is widely recognized that the demand side design is a key enabler to accomplishing the electricity distribution in a stable and energy-efficient way.

The main purpose of this paper is to propose the physical architecture of an electric power system with multiple homes. The notion of home is a unit of small-scale power system close to consumers and corresponds to an entity of the physical architecture which we propose in this paper. A home consists of local energy source, energy storage, load, power conversion circuits, and control systems. Examples of the energy source are photovoltaic (PV) array, microcombined heat and power $(\mu-\mathrm{CHP})$ plant, and fuel cell. Typical examples of the energy storage include a system of

Manuscript received January 11, 2013.

Manuscript revised April 5, 2013.

${ }^{\dagger}$ The authors are with the Department of Electrical Engineering, Kyoto University, Kyoto-shi, 615-8510 Japan. ICE-IT.

*This work was supported in part by NICT Research Project

a) E-mail: susuki.yoshihiko.5c@kyoto-u.ac.jp

b)E-mail: kazaoka@circuit.kuee.kyoto-u.ac.jp

c) E-mail: hikihara.takashi.2n@kyoto-u.ac.jp

DOI: 10.1587/transfun.E96.A.1703

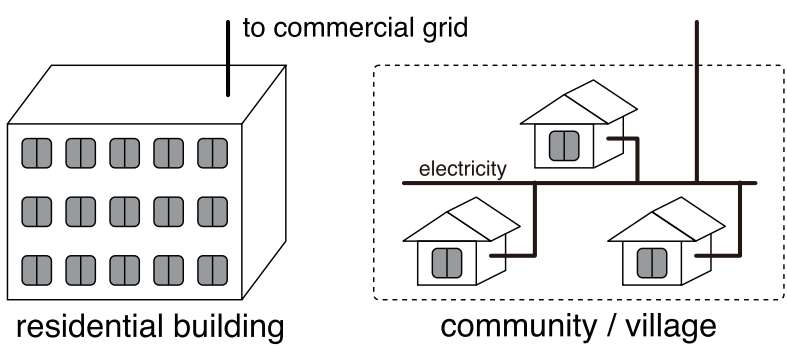

Fig. 1 Practical examples of the electric power system with multiple homes.

lithium-ion batteries and an electric vehicle connected to a household. An entire power system includes multiple homes that are interconnected via a distribution network and are connected to the commercial power grid. Practical examples of the power system are shown in Fig. 1, which include a large residential building with multiple rooms used by different consumers and a community or village with small residential buildings. The power system is related to the development of smart energy community and studied in the control engineering community [2]. Throughout this paper, we intend to perform the system-level design of small-scale power systems on the demand side.

The contributions of this paper are summarized below. First, we introduce the physical architecture of the power system with multiple homes. A key point of the architecture is to apply the technology of bi-directional, singlephase grid-connected inverters that is proposed in [3]-[5] and termed in [5] as the synchronous inverter. This application makes it possible to maintain frequency synchronization, which is a dynamical analogue of synchronous generators, and to enhance the system's stability. To the best of our knowledge, the physical architecture of small-scale power systems based on the synchronous inverter has not been reported. Second, we develop a mathematical model to represent slow dynamics of the power system. The slow dynamics correspond to time responses of frequency and active power in the mid-term regime (order of seconds to hours). The developed model enables the evaluation of static and dynamic characteristics of the system with arbitrary configuration. Lastly, we present a simple example of the modelbased evaluation in a realistic setting of parameters. The setting is based on typical commercial residential buildings and power conditioning systems in Japan. Numerical simulations of the model allow us to analyze dynamic characteristics of the power system. Also, a dynamical effect of 
uncertainty in a renewable energy source to the active power output of each home is illustrated. A preliminary version of this work was published in [6]. The present paper contains additional data of numerical simulations for the case of three homes that were not included in the preliminary work.

The rest of this paper is organized as follows. In Sect. 2 we introduce the physical architecture of the power system with multiple homes. A mathematical model to represent slow dynamics of the power system with $N$ homes is developed in Sect. 3. In Sect. 4, numerical simulations of the model are presented for the case of three $(N=3)$ homes. Section 5 is the conclusion of this paper with future directions.

\section{Physical Architecture}

This section introduces main objectives of the power system with multiple homes and its physical architecture. In the language of the monograph [7], the physical architecture is at minimum a node-arc representation of physical resources and their interconnections; also the architecture comprises entities and the structure of relationships and interfaces between them. Following these, we describe an inhome power system as the entity, an inter-home distribution network as the structure of relationships, and an interface circuit as the interface between them.

\subsection{Main Objectives}

Our target level to manage electricity is in the range of onehome to multiple-home capacities, that is, a few $\mathrm{kW}$ to a few dozen $\mathrm{kW}$. In this target level, there are three main objectives that should be achieved in the power system. The first objective is to realize new functionalities of electricity management using the information and communications technology. Examples of the functionalities include the delivery of power between different homes and the application of control mechanisms driven by market and energy-efficiency policies. The second one is to ensure the system's stability. Because the notion of stability is very broad [8], it needs to choose appropriate definitions for stability of interest. Since the first objective implies the occurrence of non-static flow in the power system, steady-state and dynamic stability of frequency and voltage should be maintained in short- and mid-terms. The third objective is to ensure the system's reliability. This includes the tasks of keeping the quality of power, of reducing the incidence of severe accidents, and of imposing the zero reverse flow to the commercial grid. For the quality task, it is possible to utilize the control ability of grid-connected inverters [9].

Note that these objectives are often imposed on the socalled Microgrid [10], [11]. The Microgrid is being developed as an entity of the conventional power grid and is a unit of small-scale power system with small energy source, storage device, and load. In comparison with this, there are several different features of the power system which we address in this paper. First, our target level of the power sys-

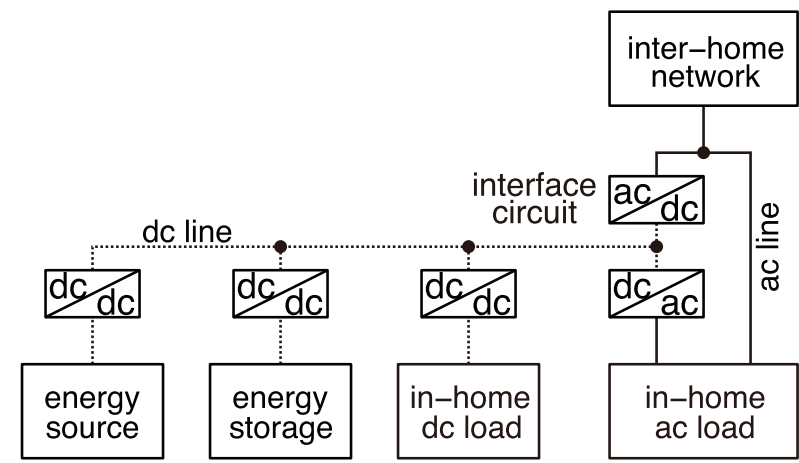

Fig. 2 Physical architecture of the in-home power system.

tem is lower than that of most Microgrid projects [11]. Second, the geographical area of the system is in the range of a few dozen homes and is narrower than that for the Microgrid projects. Third, our system is on the demand side design with emphasis on consumers in households and offices. Thus, an effective combination of these different power grid systems is expected to contribute the total efficiency in the current electricity infrastructure.

\subsection{In-Home Architecture}

Figure 2 shows a physical architecture of the in-home power system. The system consists of an energy generation unit (PV array, $\mu$-CHP, fuel cell, etc.), an energy storage unit (secondary lithium-ion battery, electric vehicle, etc.), inhome ac and dc loads, and power conversion circuits. The solid line stands for the ac electricity line, and the dotted line the dc electricity line. The dc equipment is connected on a single dc bus. The power system is connected to the outside of the home. The connection has the two ways of electricity: one is direct to the in-home ac load, and the other is the interface circuit. Here, we apply a technology of uni-directional, single-phase grid-connected inverters to the interface circuit between the in-home dc system and the ac system, which we term as the interface circuit. This technology is based on [3]-[5]. In [3], [4] the authors developed a new control method for dc/ac conversion that connected a PV array with the commercial ac grid. The control method uses a voltagecontrolled oscillator (VCO) that adjusts the frequency of inverter output and achieves frequency synchronization. Also it is shown in [3], [4] that the control method becomes an alternative of the MPPT (Maximum Power Point Tracker), which is applied in practical and commercial systems. In [5] the authors refined the control method with a phase-locked loop circuit, which they termed as the synchronous inverter. The synchronous inverter enables the mutual synchronization of frequency as well as phase and the enhancement of stability of a grid-connected system. For details of the interface circuit, see [3]-[5]. The interface circuit shown in Fig. 2 has two main roles for the management of electricity: one is the exchange of power between the in-home dc system and the ac system, and the other is the enhancement of stability in a system of interconnected homes. Note that 


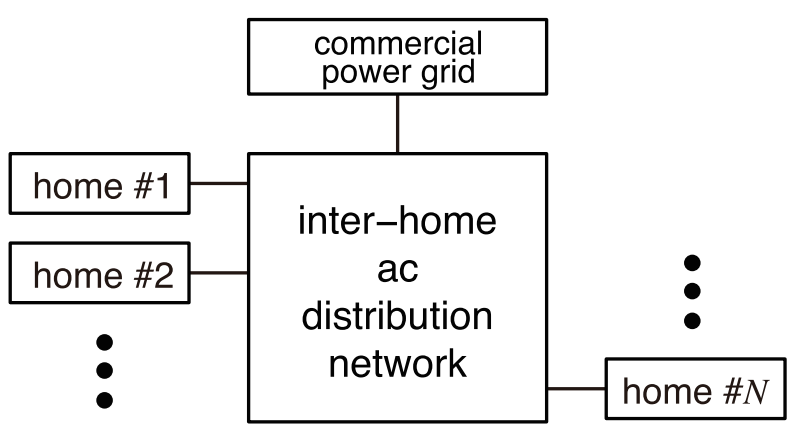

Fig. 3 Physical architecture of the electric power system with multiple homes.

similar structures of the in-home power system are reported in [12], [13] as a small-scale dc-based Microgrid.

\subsection{Inter-Home Architecture}

Figure 3 shows the physical architecture of the power system with multiple homes. The $N$ homes are interconnected locally and connected via an ac low-voltage distribution network. The whole network is connected to the commercial power grid. For the distribution network, there are several graph topologies on which the $N$ homes are dynamically interacted: ladder, ring, and star topologies. The choice of network topology affects the ability of power system as well as its stability and reliability. In Sect. 4, we will numerically study dynamics of the power system with three $(N=3)$ homes and ladder topology.

\section{Mathematical Model}

This section develops a mathematical model of the power system with $N$ homes. Here we consider a home consisting of a PV generation unit, a battery unit, an in-home dc load, and the interface circuit. This structure is a simplified one in Fig. 2, and the dc and ac loads are replaced by one dc load. This replacement is valid for the ideal operation of power conversion circuit faced on the ac load. Before modeling procedure, we make the following assumptions:

1. Voltage amplitude is regulated at a nominal value.

2. In-home distribution network is lossless.

3. Interface circuit is lossless, and its operation is ideal.

4. Inter-home distribution network consists of passive elements.

5. Battery unit is not operated, and its dynamics are negligible.

6. Every home has common equipments of the in-home power system.

7. The commercial power grid is modeled with an infinite bus [14].

All the assumptions except for Assumption 5 are reasonable for developing the mathematical model of slow dynamics in the power system, whose time scale is much larger than the scales of the nominal alternative signal (namely, $16.7 \mathrm{~ms}$

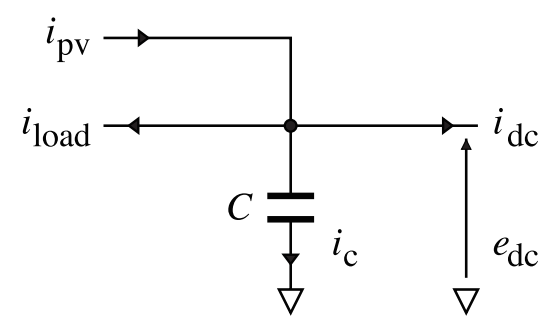

Fig. 4 Current variables defined in the in-home power system without battery.

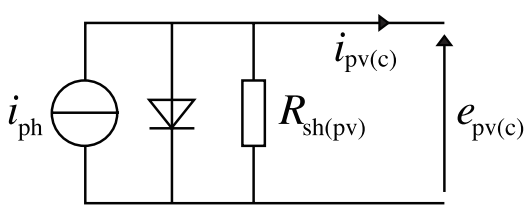

Fig. 5 Simple circuit model of a photovoltaic (PV) cell.

or $20 \mathrm{~ms}$ ). In this way, no detailed model of converter dynamics and control of PV generation unit are needed. Also, from Assumption 1, we model active power flows in the system and do not need to consider reactive power flows determining the voltage amplitude in the system. Assumption 5 implies no battery control in the power system and may be crucial to the objectives of the power system. The goal of this section is to model nominal slow dynamics of the power system.

Here, the basic idea of mathematical modeling for the dynamics of power system is described. For each component in a home, we provide a mathematical description of the relationship between its output current and the dc system voltage $e_{\mathrm{dc}}$. The normalized PV output current, load input current, and dc current are denoted by $i_{\mathrm{pv}}, i_{\text {load }}$, and $i_{\mathrm{dc}}$ : see Fig. 4 . The normalized capacitor current with a constant capacitance $C$ is also denoted by $i_{\mathrm{c}}$. The capacitance is equipped with the interface circuit to smooth the dc voltage. In Fig. 4, the Kirchhoff's current law gives the following equality: at any moment $t$,

$$
i_{\mathrm{pv}}(t)=i_{\text {load }}(t)+i_{\mathrm{dc}}(t)+i_{\mathrm{c}}(t) .
$$

The relationship between $e_{\mathrm{dc}}$ and $i_{\mathrm{c}}$ is simply the following:

$$
C \frac{\mathrm{d} e_{\mathrm{dc}}}{\mathrm{d} t}=i_{\mathrm{c}} .
$$

In this way, by combing these equations, the mathematical model of the dynamics of power system is derived.

\subsection{PV Generation Unit}

Figure 5 shows the simple circuit model of a PV cell that we use in this paper. This circuit model consists of the ideal dc current source $i_{\mathrm{ph}}$, the ideal diode, and the shunt resistance $R_{\mathrm{sh}(\mathrm{pv})}$. The parameter $i_{\mathrm{ph}}$ is the photoelectronic current produced by light emission. In this model, the following relationship between the cell output current $i_{\mathrm{pv}(\mathrm{c})}$ and the cell terminal voltage $e_{\mathrm{pv}(\mathrm{c})}$ holds: 


$$
i_{\mathrm{ph}}=I_{0}\left\{\exp \left(\frac{e_{\mathrm{pv}(\mathrm{c})}}{V_{T}}\right)-1\right\}+\frac{e_{\mathrm{pv}(\mathrm{c})}}{R_{\mathrm{sh}(\mathrm{pv})}}+i_{\mathrm{pv}(\mathrm{c})},
$$

where $I_{0}$ is the reverse bias saturation current, and $V_{T}$ is the thermal voltage at temperature $T$ in Kelvin. Here, let us regard $i_{\mathrm{pv}}$ as the output current of a PV array that consists of $N_{\mathrm{s}}$ cells in series and $N_{\mathrm{p}}$ cells in parallel. The current $i_{\mathrm{pv}}$ is thus represented as

$$
\begin{aligned}
i_{\mathrm{pv}}= & N_{\mathrm{p}} i_{\mathrm{pv}(\mathrm{c})} \\
= & N_{\mathrm{p}}\left[i_{\mathrm{ph}}-I_{0}\left\{\exp \left(\frac{e_{\mathrm{dc}}}{N_{\mathrm{s}} V_{T}}\right)-1\right\}\right. \\
& \left.-\frac{e_{\mathrm{dc}}}{N_{\mathrm{s}} R_{\mathrm{sh}(\mathrm{pv})}}\right],
\end{aligned}
$$

where the direct relation $e_{\mathrm{pv}(\mathrm{c})}=e_{\mathrm{dc}} / N_{\mathrm{s}}$ is used under an additional assumption of the PV array in which each cell has a common value of terminal voltage.

\subsection{In-Home DC Load}

Simply, we consider the in-home load as a constant power load $p_{\text {load }}$. Then, the load input current $i_{\text {load }}$ is represented as

$$
i_{\text {load }}=\frac{p_{\text {load }}}{e_{\mathrm{dc}}} .
$$

\subsection{Interface Circuit}

Basically, we use the mathematical model of the interface circuit presented in [3], [5], but we improve it slightly in order to represent the dynamical interaction of multiple homes. Figure 6 shows the block diagram of the VCO that produces the PWM reference signal. The mathematical model of the VCO is simply the following:

$$
\omega_{\mathrm{inv}}=\frac{\omega_{0}}{E_{0}} e_{\mathrm{dc}}=K\left(e_{\mathrm{dc}}-E_{0}\right)+\omega_{0},
$$

where $\omega_{0}$ is the nominal angular frequency, $E_{0}$ is the nominal value of $e_{\mathrm{dc}}$, and $K:=\omega_{0} / E_{0}$ is the gain constant for frequency regulation. By defining the new variable $\Delta \omega$ as $\omega_{\text {inv }}-\omega_{0}$, we have

$$
\Delta \omega=K\left(e_{\mathrm{dc}}-E_{0}\right) .
$$

Here, it follows from Assumption 3 that the active power balance between the dc and ac systems satisfies

$$
e_{\mathrm{dc}} i_{\mathrm{dc}}=\operatorname{Re}\left[\dot{E} \dot{I}^{*}\right]=: p,
$$

where $\dot{E}$ (or $\dot{I}$ ) stands for the ac output voltage (or current) of the interface circuit in phasor, and the symbol $*$ for

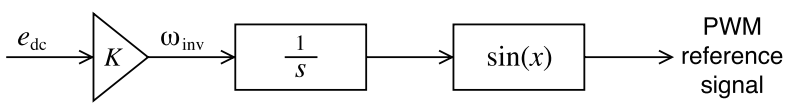

Fig. 6 Block diagram of the voltage-controlled oscillator (VCO) that produces the PWM reference signal. the conjugate operation of complex variables. The variable $\delta$ is termed as the phase angle in literature of power systems engineering [14]. By combining Eqs. (1), (2), (7), and (8), the following differential equations to represent the time changes of $\delta$ and $\Delta \omega$ are derived:

$$
\frac{\mathrm{d} \delta}{\mathrm{d} t}=\Delta \omega, \quad \frac{\mathrm{d} \Delta \omega}{\mathrm{d} t}=\frac{K}{C}\left(i_{\mathrm{pv}}-i_{\mathrm{load}}-\frac{p}{e_{\mathrm{dc}}}\right),
$$

where recall that $e_{\mathrm{dc}}$ is a function of $\Delta \omega$. The term $p$ of the active power output from a single home is derived in the next sub-section.

\subsection{Inter-Home Distribution Network}

Throught this section, the dynamics of frequency and active power have been addressed. For this, the inter-home distribution network is modeled in a framework of quasistatic phasor representation [14]. For given cutset matrix $C$ and branch admittance matrix $Y_{\text {branch }}$ of the distribution network, the admittance matrix $Y$ of the distribution network becomes $\mathrm{CY}_{\text {branch }} \mathrm{C}^{\top}$. The symbol $\mathrm{T}$ stands for the transpose operation of vectors and matrices. Thus, the standard current-voltage relation $\dot{\boldsymbol{I}}=\mathrm{Y} \dot{\boldsymbol{V}}$ holds, where $\dot{I}:=\left(\dot{I}_{1}, \dot{I}_{2}, \ldots, \dot{I}_{N}, \dot{I}_{N+1}\right)^{\top}$ is the output current vector of the $N$ interface circuits and the commercial grid $(N+1)$, and $\dot{\boldsymbol{V}}:=\left(\dot{V}_{1}, \dot{V}_{2}, \ldots, \dot{V}_{N}, \dot{V}_{N+1}\right)^{\top}$ is the voltage vector at the linkage points of the $N$ interface circuits and the commercial grid. What we now need is the relationship between $\dot{\boldsymbol{I}}$ and the output voltage vectors $\dot{\boldsymbol{E}}:=\left(\dot{E}_{1}, \dot{E}_{2}, \ldots, \dot{E}_{N}, \dot{E}_{N+1}\right)^{\top}$ of the $N$ interface circuits and the commercial grid. Here, by using $\dot{Z}_{\text {link }}$ to represent the vector of linkage impedance from each home to the network, we have

$$
\dot{\boldsymbol{E}}=\operatorname{diag}\left(\dot{\boldsymbol{Z}}_{\text {link }}\right) \dot{\boldsymbol{I}}+\dot{\boldsymbol{V}},
$$

where $\operatorname{diag}(\cdot)$ stands for the diagonal matrix made from a vector. By using $\dot{\boldsymbol{I}}=\mathrm{Y} \dot{\boldsymbol{V}}$, the following relation between $\dot{\boldsymbol{I}}$ and $\dot{\boldsymbol{E}}$ holds:

$$
\dot{\boldsymbol{I}}=(\mathrm{G}+\mathrm{jB}) \dot{\boldsymbol{E}},
$$

with

$$
\mathrm{G}+\mathrm{jB}:=\left(\mathrm{Y} \operatorname{diag}\left(\dot{\mathrm{Z}}_{\text {link }}\right)+\mathrm{I}_{N+1}\right)^{-1} \mathrm{Y},
$$

where $\mathrm{I}_{N+1}$ stands for the identity matrix of size $N+1$. Both $G$ and $B$ are real-valued, symmetric matrices with constant entries. The relation (11) is used for the calculation of the term $p_{k}$ of active output power from home \#k $(k=1,2, \ldots, N)$ :

$$
\begin{aligned}
p_{k}:= & \operatorname{Re}\left[\dot{E}_{k} \dot{I}_{k}^{*}\right] \\
= & G_{k k} E_{k}^{2}+\sum_{l=1, l \neq k}^{N+1} E_{k} E_{l}\left\{G_{k l} \cos \left(\delta_{k}-\delta_{l}\right)\right. \\
& \left.+B_{k l} \sin \left(\delta_{k}-\delta_{l}\right)\right\},
\end{aligned}
$$

where $G_{k l}$ stands for the matrix entry in the $k$-th row and $l$-th column. This is the reason why the term $p_{k}$ is a function of 
Table 1 List of parameters of the electric power system with three homes.

\begin{tabular}{|c|c|c|c|}
\hline Symbol & Meaning & $\begin{array}{c}\text { Value fixed } \\
\text { for simulation }\end{array}$ & $\begin{array}{c}\text { Normalized } \\
\text { Value }\end{array}$ \\
\hline$R_{\mathrm{sh}(\mathrm{pv})}$ & Internal shunt resistance in the PV cell model & $10 \Omega$ & 0.439 \\
\hline$I_{0}$ & Reverse bias saturation current of the PV cell model & $1.0 \times 10^{-15} \mathrm{~A}$ & $6.17 \times 10^{-17}$ \\
\hline$q$ & Elementary charge & \multicolumn{2}{|c|}{$1.602176487(40) \times 10^{-19} \mathrm{C}$} \\
\hline$k_{\mathrm{B}}$ & Boltzmann constant & \multicolumn{2}{|c|}{$1.3806504(24) \times 10^{-23} \mathrm{~J} / \mathrm{K}$} \\
\hline$T$ & Temperature (in Kelvin) & $300 \mathrm{~K}$ & \\
\hline$V_{T}$ & Thermal voltage $k_{\mathrm{B}} T / q$ & $0.0258 \mathrm{~V}$ & $6.97 \times 10^{-5}$ \\
\hline$N_{\mathrm{S}}$ & Number of the PV cells connected in a series way & 480 & 480 \\
\hline$N_{\mathrm{p}}$ & Number of the PV cells connected in a parallel way & 20 & 20 \\
\hline$p_{\text {load }, k}$ & Power consumed in the load of home \#k & & (in text) \\
\hline$\delta_{4}$ & Phase angle of the commercial power grid (infinite bus) & 0 & 0 \\
\hline$\omega_{0}$ & Nominal angular frequency & $2 \pi \times 60 \mathrm{~Hz}$ & 1 \\
\hline$E_{0}$ & Nominal value of the dc system voltage $e_{\mathrm{dc}, k}$ in home \#k & $370 \mathrm{~V}$ & 1 \\
\hline$K$ & Gain constant of the voltage-controlled oscillator & $1.109 \mathrm{rad} / \mathrm{s} / \mathrm{V}$ & 1 \\
\hline$C$ & Smoothing capacitance in the interface circuit & $10 \mathrm{mF}$ & 85.95 \\
\hline$R+\mathrm{j} X$ & Line impedance between nearest neighbor homes & $\begin{array}{l}3.0 \times 10^{-3} \Omega(R) \\
4.0 \times 10^{-3} \Omega(X)\end{array}$ & $\begin{array}{l}4.5 \times 10^{-4} \\
6.0 \times 10^{-4}\end{array}$ \\
\hline$R_{\infty}+\mathrm{j} X_{\infty}$ & Line impedance connected to the commercial power grid & $\begin{array}{l}3.0 \times 10^{-2} \Omega\left(R_{\infty}\right) \\
4.0 \times 10^{-2} \Omega\left(X_{\infty}\right)\end{array}$ & $\begin{array}{l}4.5 \times 10^{-3} \\
6.0 \times 10^{-3}\end{array}$ \\
\hline$X_{\mathrm{T}}$ & Transformer reactance & & 0.06 \\
\hline$X_{\mathrm{L}}$ & Linkage reactance between a home and the distribution network & & 0.02 \\
\hline
\end{tabular}

phase angles $\delta_{k}$ in all the homes.

\subsection{Full Model}

Consequently, the following full model to represent the dynamics of frequency and active power in the $N$ homes is derived: for home $\# k(k=1, \ldots, N)$,

$$
\left.\begin{array}{rl}
\frac{\mathrm{d} \delta_{k}}{\mathrm{~d} t}= & \Delta \omega_{k}, \\
\frac{\mathrm{d} \Delta \omega_{k}}{\mathrm{~d} t}= & \frac{K}{C}\left(i_{\mathrm{pv}, k}-\frac{p_{\text {load }, k}}{e_{\mathrm{dc}, k}}-\frac{p_{k}}{e_{\mathrm{dc}, k}}\right), \\
e_{\mathrm{dc}, k} & =E_{0}+\frac{\Delta \omega_{k}}{K}, \\
i_{\mathrm{pv}, k}= & N_{\mathrm{p}}\left[i_{\mathrm{ph}, k}-I_{0}\left\{\exp \left(\frac{e_{\mathrm{dc}, k}}{N_{\mathrm{s}} V_{T}}\right)-1\right\}\right. \\
& \left.-\frac{e_{\mathrm{dc}, k}}{N_{\mathrm{s}} R_{\mathrm{sh}(\mathrm{pv})}}\right],
\end{array}\right\}
$$

where the term $p_{k}$ of the active power output is presented in Eq. (13).

Because of the design of interface circuit, we show that the full model (14) has a coupling structure similar to that in the multi degree-of-freedom swing equations for transient stability analysis of multi-machine power grids [14]-[16]. Under the parameter setting in Sect. 4, the model (14) has a particular structure of neighbor coupling and long-range effect, which has been extensively studied in [16]-[19]. However, by comparison with [17]-[19], there is one different feature of the model (14) in which the anti-symmetric coupling term, $G_{k l} \cos \left(\delta_{k}-\delta_{l}\right)$, exists. The term comes from a non-zero resistive element in the inter-home distribution network, whose value is compatible with the reactance element in a low-voltage line: see Table 1. In [20] the author proves that there is no energy function for a multi degreeof-freedom swing equation if its degree is greater than or equal to two and it has an anti-symmetric coupling term like above. The existence of energy function implies no (bounded) oscillatory attractor in the swing equation. In this way, we theoretically suggest that the slow dynamics described by the model (14) are likely non-integrable.

\section{Example of Model-Based Evaluation}

The model developed in Sect. 3 is applicable to the evaluation of steady and dynamic characteristics of a power system. In this section, as a simple example of the model-based evaluation, we perform numerical simulations of Eq. (14) in the case of three $(N=3)$ homes. The corresponding power system is shown in Fig. 7. Since the current model does not fully include ancillary control systems such as flow management, the associated dynamics are regarded as an uncontrolled pure case. Analyzing the pure case allows us to define nominal dynamics of the power system that are regulated by control. Equation (14) possesses many uncertain parameters that include the injection current $i_{\mathrm{pv}, k}$ of the PV array and the load profile $p_{\text {load }, k}$. We numerically investigate the dynamics of the system under a change of the injection current $i_{\mathrm{pv}, k}$. The values of parameters for the three homes are given in Table 1. The normalized values in the right column of Table 1 are used for numerical simulations. The normalization is performed with the base quantities of variables and parameters shown in Table 2.

\subsection{Modeling of a Ladder Network}

For numerical simulations, it needs to compute the conductance matrix $G$ and the susceptance matrix $B$. Consider the inter-home distribution network with the ladder topology shown in Fig. 8. The parameters $R, X, R_{\infty}, X_{\infty}, R_{\mathrm{L}}, X_{\mathrm{L}}$, and $X_{\mathrm{T}}$ are constant and given in Table 1 . For the network, the matrices $C$ and $\mathrm{Y}_{\text {branch }}$, and the vector $\dot{Z}_{\text {link }}$ are obtained 


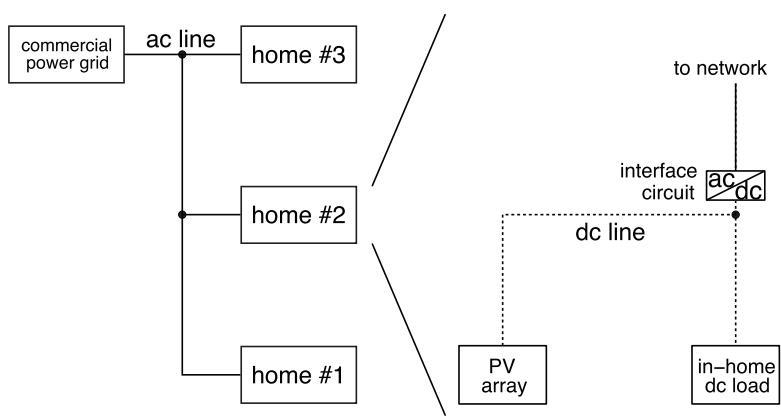

Fig. 7 Configuration of the electric power system with three homes considered in this paper.

Table 2 Bese quantities.

\begin{tabular}{cccc}
\hline Symbol & In-Home DC & In-Home AC & Inter-Home \\
\hline$W_{\text {base }}$ & $6 \mathrm{~kW}$ & $6 \mathrm{kVA}$ & $6 \mathrm{kVA}$ \\
$V_{\text {base }}$ & $370 \mathrm{~V}$ & $200 \mathrm{~V}_{\mathrm{rms}}$ & $6600 \mathrm{~V}_{\mathrm{rms}}$ \\
$I_{\text {base }}$ & $16.2 \mathrm{~A}$ & $30 \mathrm{~A}_{\mathrm{rms}}$ & $0.909 \mathrm{~A}_{\mathrm{rms}}$ \\
$Z_{\text {base }}$ & $22.8 \Omega$ & $6.66 \Omega$ & $7.26 \times 10^{3} \Omega$ \\
$\omega_{\text {base }}$ & $2 \pi \times 60 \mathrm{rad} / \mathrm{s}$ & $2 \pi \times 60 \mathrm{rad} / \mathrm{s}$ & $2 \pi \times 60 \mathrm{rad} / \mathrm{s}$ \\
\hline
\end{tabular}

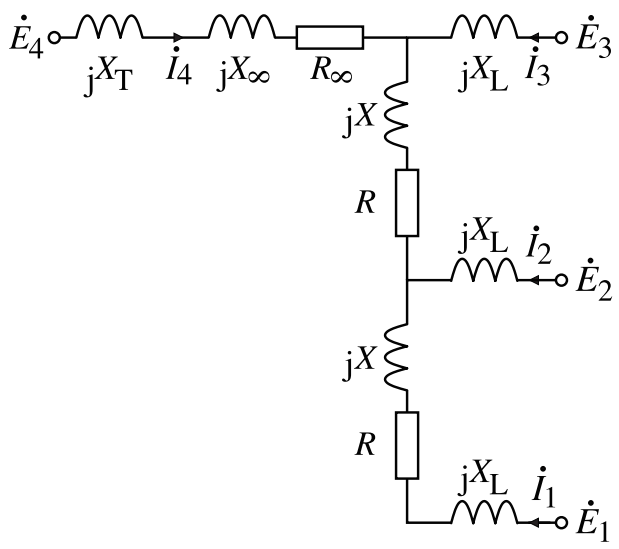

Fig. 8 Inter-home distribution network for the electric power system with three homes in Fig. 7.

as follows:

$$
\begin{aligned}
\mathrm{C}= & \left(\begin{array}{rrr}
1 & 0 & 0 \\
-1 & 1 & 0 \\
0 & -1 & 1 \\
0 & 0 & -1
\end{array}\right), \\
\mathrm{Y}_{\text {branch }}= & \operatorname{diag}\left(\left((R+\mathrm{j} X)^{-1},(R+\mathrm{j} X)^{-1},\right.\right. \\
& \left.\left.\left(R_{\infty}+\mathrm{j} X_{\infty}\right)^{-1}\right)^{\top}\right), \\
\mathrm{Y} \quad= & \mathrm{CY}_{\text {branch }} \mathrm{C}^{\top}, \\
\dot{Z}_{\text {link }}= & \mathrm{j}\left(X_{\mathrm{L}}, X_{\mathrm{L}}, X_{\mathrm{L}}, X_{\mathrm{T}}\right)^{\top} .
\end{aligned}
$$

With these and Eq. (12), the two matrices $G$ and B are computed.

\subsection{Steady-State Characteristics}

First, let us consider steady-state characteristics of the three homes. Figure 9(i) shows the values of phase angles $\delta_{k}$ under equilibriums with the change of the photoelectric cur-
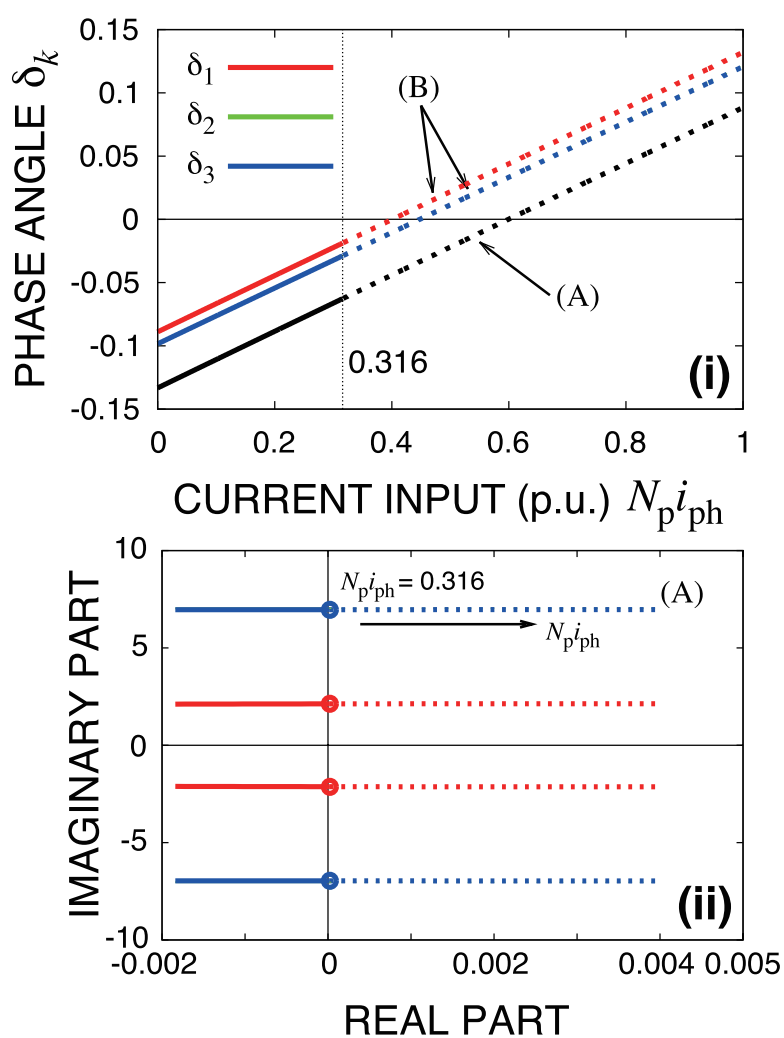

Fig. 9 (i) Change of the values of phase angles $\delta_{k}$ under equilibriums and (ii) loci of their (linearized) eigenvalues around the bifurcation value: (A) uniform load profile and (B) non-uniform load profile. The solid lines represent the stable equilibriums, and the dotted lines the unstable equilibriums.

rent $N_{\mathrm{p}} i_{\mathrm{ph}}$. The two cases of uniform and non-uniform load profiles are now considered: (A) $\left(p_{\text {load }, 1}, p_{\text {load }, 2}, p_{\text {load }, 3}\right)=$ $(0.5,0.5,0.5)$ and $(\mathrm{B})\left(p_{\text {load }, 1}, p_{\text {load }, 2}, p_{\text {load }, 3}\right)=(0,0.5,0.5)$. For this analysis, we assume the uniform photoelectric current for every home, that is, $i_{\mathrm{ph}, k}=i_{\mathrm{ph}}$. This uniform setting of $i_{\mathrm{ph}}$ is tractable if the geographical area covering the three homes is small. In the uniform profile (A), all the values of phase angles $\delta_{i}$ are the same. The non-uniform profile (B) results in non-uniform equilibrium values of $\delta_{k}$ as shown in Fig. 9(i). The values of the two phase angles $\delta_{2}$ and $\delta_{3}$, denoted by the blue line in Fig. 9(i), are the same and are different from the value of $\delta_{1}$, denoted by the red line. In this figure, the solid lines represent the stable equilibriums, and the dotted lines the unstable equilibriums. Thus, the stability of the equilibriums changes at $N_{\mathrm{p}} i_{\mathrm{ph}}=0.316$. By linearization of Eq. (14) around the equilibriums, the change of stability is due to supercritical Hopf bifurcation [21] as shown in Fig. 9(ii) for the case (A). After the bifurcation value, a stable limit cycle is generated around the equilibrium. The stable limit cycle implies undamped, sustained oscillations in frequency and active power, and it is regarded as a undesirable operating condition of the power system. The existence of limit cycle is an evidence of the non-integrablity of the dynamical system model (14) which is mentioned in Sect. 3.5. 
(i)

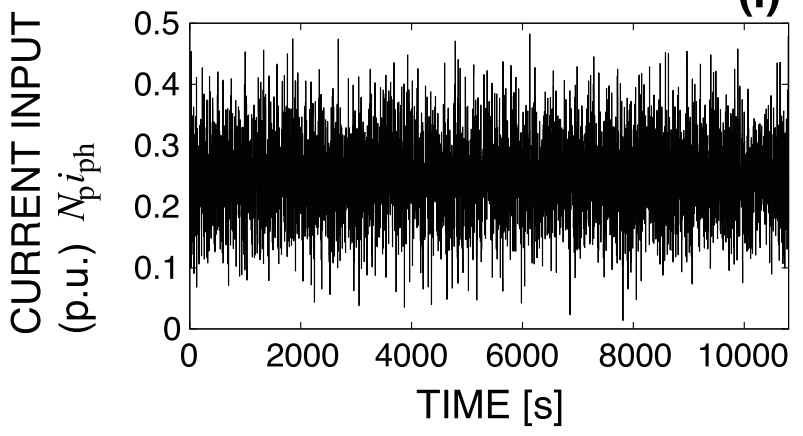

(ii)

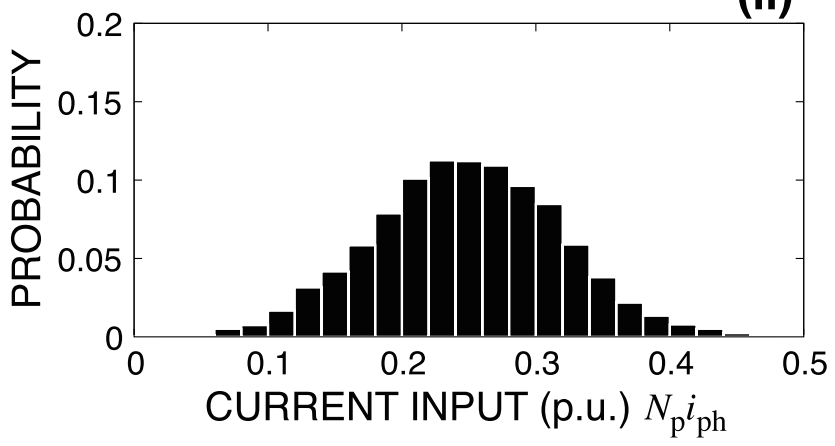

Fig. 10 (i) Time series and (ii) probability density of uncertain input from the photoelectric current of the PV array used in this paper.

(i)

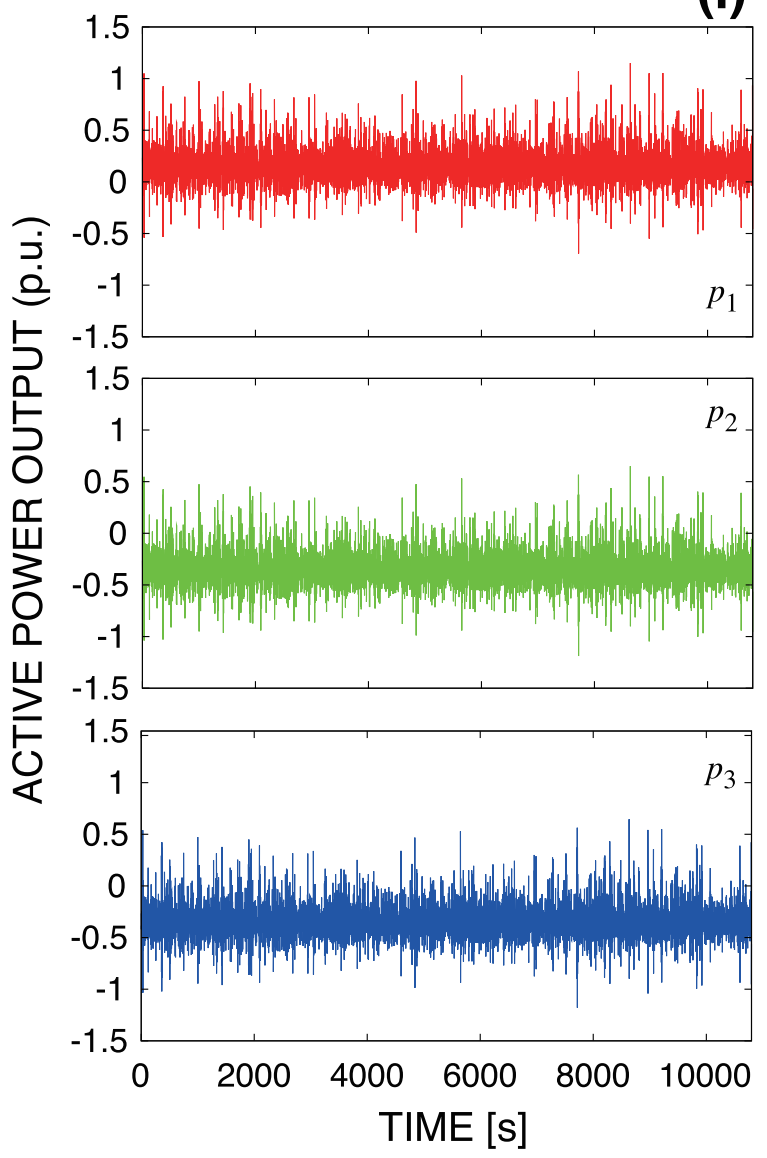

(ii)
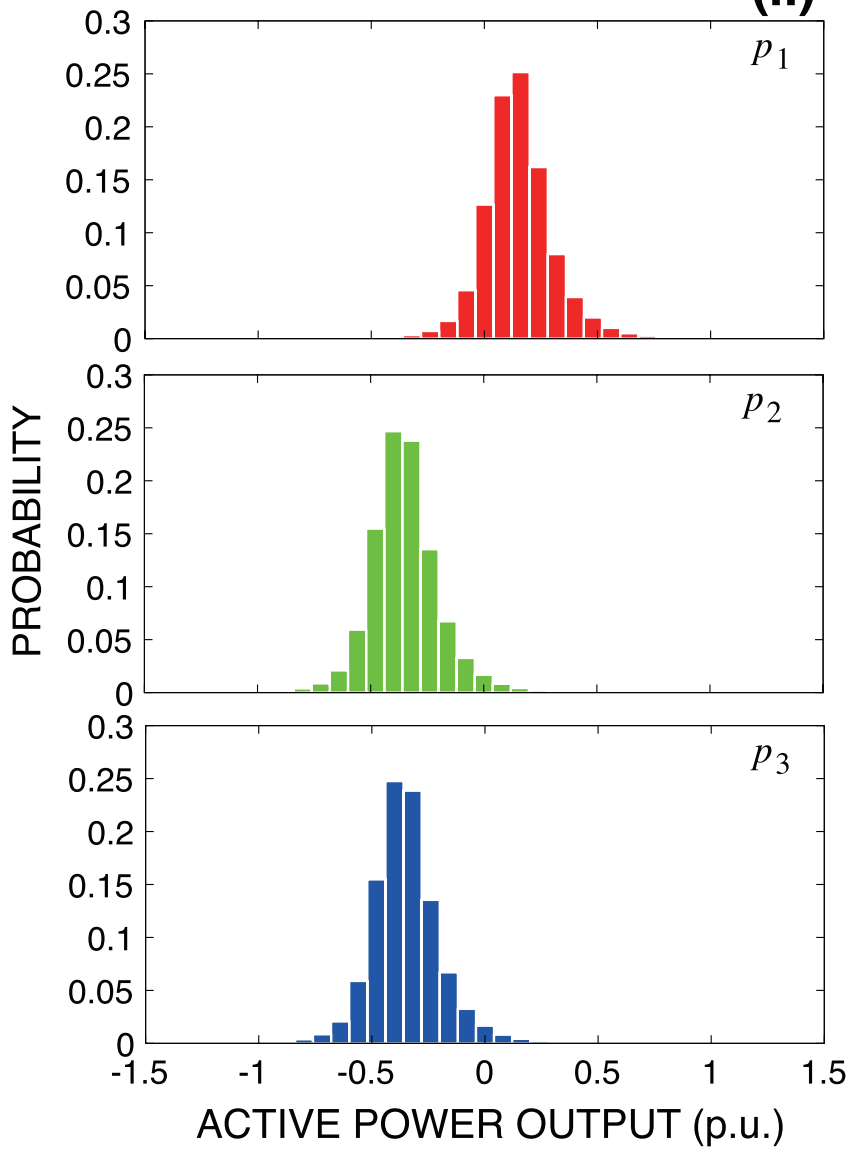

Fig. 11 (i) Time series and (ii) probability densities of the active power output $p_{k}$ while the photoelectric current $N_{\mathrm{p}} i_{\mathrm{ph}}$ changes in a probabilistic way. This is the case of non-uniform load profile (A).

\subsection{Long-Term Dynamic Characteristics}

Next, we discuss long-term dynamic characteristics caused by a combination of the bifurcation phenomenon and uncertain change of the photoelectric current. As mentioned above, the parameter $N_{\mathrm{p}} i_{\mathrm{ph}}$ is uncertain and is modeled in a probabilistic way. One simple example of such time series of $N_{\mathrm{p}} i_{\mathrm{ph}}$ is shown in Fig. 10(i). The value of $N_{\mathrm{p}} i_{\mathrm{ph}}$ changes at every 5 seconds. The time series is generated as a Gaussian distribution with mean 0.25 and standard deviation 0.071 [22]. Figure 10(ii) shows the probability density of the time series. The time series are ideal in the sense that over the time dime duration of Fig. 10(i) the mean value of the solar irradiance is expected to change; moreover, the deviation from the mean value is too big. The density is computed 
by counting the time spent for each $N_{\mathrm{p}} i_{\mathrm{ph}}$ and dividing it by the total time duration in Fig. 10(i). This time series is regarded as an uncertain input to the dynamical system (14). This viewpoint of the dynamical system comes from the idea of uncertainty propagation in nonlinear systems [23]. Figure 11 shows the long-term responses of the active power output $p_{k}$ against the time series of $N_{\mathrm{p}} i_{\mathrm{ph}}$. This is the case of non-uniform load profile (B). The probability densities of the active power output are also shown. Intermittent behaviors are observed in the course of long-term dynamics that do not appear in the time series of $N_{\mathrm{p}} i_{\mathrm{ph}}$. The behaviors occur due to a combination of the Hopf bifurcation and the uncertain input. That is, while the value of $N_{\mathrm{p}} i_{\mathrm{ph}}$ is larger than the bifurcation value, the dynamics do not settle down to any equilibrium and grow as times goes on. On the other hand, while $N_{\mathrm{p}} i_{\mathrm{ph}}$ is smaller than the bifurcation value, the dynamics tend to converge to a stable equilibrium. Note that a mechanism similar to this is proposed in [24].

This result suggests a possibility of complex behaviors of electric power systems due to a combination of uncertain renewables and nonlinear nominal dynamics. The stability of power systems is a well-established subject with a long history of research [15], [25]. Many phenomena of loss of stability are classified according to the mechanisms: local bifurcations due to the change of system parameters and global dynamics described by a nonlinear dynamical system far from a steady state condition [26]. The mechanisms have been mainly validated for large-scale power grids with dominant inertia and deterministic sources and loads, which are obtained via aggregation or averaging in space and time. However, these are not directly applied to small-scale power grids with stochastic sources like the power system studied in this paper, because less inertia and stochastic effects are crucial in such systems. In fact, under the condition of less inertia, a new type of instability is reported in multi-machine power grids [16]. The numerical result above is due to a combination of the Hopf bifurcation and the uncertain input. The phenomenon exhibits the intermittent behaviors which are not observed in the uncertain input and which imply a short-term amplification of oscillations in active power. The Hopf bifurcation is widely reported in nominal dynamics of power grids and power electronics: see e.g. [27]. We contend that the result provides a new mechanism of complex behaviors and instability of small-scale power systems with less inertia and stochastic sources.

\section{Conclusions}

In this paper, we reported the physical architecture of an electric power system with multiple homes and developed a mathematical model to represent slow dynamics emerging in the system. The developed model enables the evaluation of static and dynamic characteristics of the system with arbitrary configuration. Numerical simulations for the case of three homes were presented as a simple example of the model-based evaluation. The occurrence of supercritical Hopf bifurcation and associated sustained oscillations in frequency and active power were identified. We indicate that due to these nonlinear phenomena, the slow dynamics of the power system are affected by the uncertain input of the PV array in a complicated manner. Future directions of the work are as follows: (i) validation of the proposed architecture and model in practice; (ii) further evaluation of other configurations of the system, including analysis of the long-term dynamics using practical data and other uncertain parameters such as home loads (note that over the time duration of Fig. 10(i) a home load is expected to change); (iii) mathematical modeling of battery dynamics and its control method. The work reported in this paper helps to develop the demand side management of electricity on the level of multiple homes.

\section{Acknowledgement}

We are grateful to Dr. Ryo Takahashi (Kyoto University) for his valuable comments.

\section{References}

[1] A. Ipakchi and F. Albuyeh, "Grid of the future," IEEE Power Energy Mag., vol.7, no.2, no.2, pp.52-62, March-April 2009.

[2] G.K.H. Larsen, J.M.A. Scherpen, N.D. van Foreest, and E. Doornbos, "Distributed control in a network of households with $\mu$ CHP," Preprint of the 18th IFAC World Congress, pp.5320-5325, 2011.

[3] K. Harada and K. Murata, "Interface circuit between solarcell and commercial ac bus," IEICE Trans. Commun. (Japanese Edition), vol.J69-C, no.11, pp.1458-1464, Nov. 1986.

[4] K. Harada and K. Murata, "On the automatic interconnection between solar cell and ac power source," Proc. Telecommunications Energy Conference, pp.259-262, 1984.

[5] T. Hikihara, T. Sawada, and T. Funaki, "Enhanced entrainment of synchronous inverters for distributed power sources," IEICE Trans. Fundamentals, vol.E90-A, no.11, pp.2516-2525, Nov. 2007.

[6] Y. Susuki, R. Kazaoka, and T. Hikihara, "Physical architectures and mathematical models for electric-power management of multiple homes," Proc. 50th IEEE Conference on Decision and Control and European Control Conference, pp.7703-7710, Dec. 2011.

[7] The Influence of Architecture in Engineering Systems, in Engineering Systems Monograph, Prepared by the MIT Engineering Systems Division, March 2004 (available at http://esd.mit.edu/symposium/ monograph/)

[8] IEEE/CIGRE Joint Task Force on Stability Terms and Definitions, "Definition and classification of power system stability," IEEE Trans. Power Syst., vol.19, no.2, pp.1387-1401, May 2004.

[9] S.B. Kjaer, J.K. Pedersen, and F. Blaabjerg, "A review of singlephase grid connected inverters for photovoltaic modules," IEEE Trans. Ind. Appl., vol.41, pp.1292-1306, Sept./Oct. 2005.

[10] B. Lasseter, "Microgrids," IEEE PES Winter Meeting Panel, pp.146-149, 2001

[11] N. Hatziargyriou, H. Asano, R. Iravani, and C. Marnay, "Microgrids," IEEE Power Energy Mag., vol.5, no.4, pp.78-94, July/Aug. 2007.

[12] B.T. Patterson, "DC, come home," IEEE Power Energy Mag., vol.10, no.6, pp.60-69, Nov.-Dec. 2012.

[13] D. Wang and F.Z. Peng, "Smart gateway grid: A DG-based residential electric power supply system," IEEE Trans. Smart Grids, 2012. (online published).

[14] P. Kundur, Power System Stability and Control, McGraw-Hill, 1994.

[15] H.D. Chiang, "Power system stability," in Wiley Encyclopedia of 
Electrical and Electronics Engineering, ed. J.G. Webster, pp.105137, John Wiley \& Sons, New York, 1999.

[16] Y. Susuki, I. Mezić, and T. Hikihara, "Coherent swing instability of power grids," J. Nonlinear Sci., vol.21, no.3, pp.403-439, June 2011.

[17] I. Mezić, "On the dynamics of molecular conformation," Natl. Acad. Sci. USA, vol.103, no.20, pp.7542-7547, 2006.

[18] P. Du Toit, I. Mezić, and J. Marsden, "Coupled oscillator models with no scale separation," Physica D, vol.238, no.5, pp.490-501, March 2009.

[19] B. Eisenhower and I. Mezić, "Targeted activation in deterministic and stochastic systems," Phys. Rev. E, vol.81, 026603, Feb. 2010.

[20] H.D. Chiang, "Study of the existence of energy functions for power systems with losses," IEEE Trans. Circuits Syst., vol.36, no.11, pp.1423-1429, Nov. 1989.

[21] J. Guckenheimer and P. Holmes, Nonlinear Oscillations, Dynamical Systems, and Bifurcations of Vector Fields, Applied Mathematical Sciences, vol.42, Springer-Verlag, New York, 1983.

[22] K.J. Åström, Introduction to Stochastic Control Theory, Dover Publications, New York, 2006

[23] I. Mezić and T. Runolfsson, "Uncertainty propagation in dynamical systems," Automatica, vol.44, pp.3003-3013, Nov. 2006.

[24] C. Li, L. Chen, and K. Aihara, "Transient resetting: A novel mechanism for syrchrony and its biological examples," PLoS Comput. Biol., vol.2, no.8, e103, Aug. 2006.

[25] E.W. Kimbark, Power System Stability, John Wiley \& Sons, New York, 1947.

[26] "Special Issue on Nonlinear Phenomena in Power Systems: Theory anf Practical Implications," Proc. IEEE, vol.83, no.11, pp.15301587, Nov. 1995

[27] E.H. Abed and P.P. Varaiya, "Nonlinear oscillations in power systems," Electrical Power \& Energy Systems, vol.6, no.1, pp.37-43, Jan. 1984.

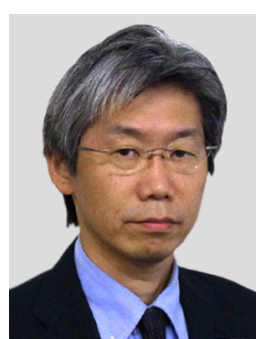

Takashi Hikihara received Ph.D. degree in Electrical Engineering from Kyoto University, Kyoto, Japan in 1990. He is currently a professor of Department of Electrical Engineering in Kyoto University. His research interests include analysis of nonlinear system, applications and control of nonlinear dynamics, power electronics, and MEMS. He is also a regular member of the IEEE, APS, SIAM, AIAA, IEEJ, and ISICE $\mathrm{He}$ is currently Fellow of IEICE and surves as the President, IEICE-ESS.

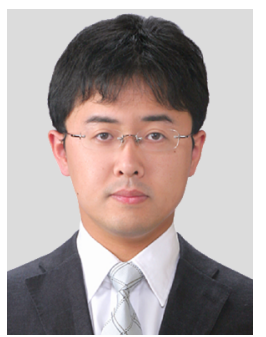

Yoshihiko Susuki received his bachelor, master, and $\mathrm{Ph} . \mathrm{D}$. degrees, all in engineering from Kyoto University, Kyoto, Japan, in 2000, 2002, and 2005, respectively. In 2005, he joined the Department of Electrical Engineering at Kyoto University, where he is currently a Lecturer. In 2008-2010, he was a Visiting Researcher at the Department of Mechanical Engineering, University of California, Santa Barbara, United States under JSPS Postdoctoral Fellowship for Research Abroad. His research interests are in power and energy engineering, nonlinear dynamical systems, and control systems. He is a member of IEEJ, ISCIE, SICE, IEEE, SIAM, and so on.

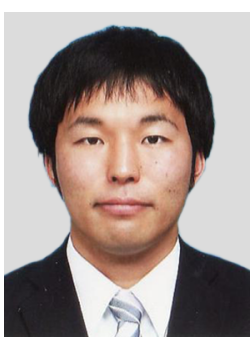

Ryoya Kazaoka received his bachelor and master degrees, all in engineering from Kyoto University, Kyoto, Japan, in 2011 and 2013, respectively. 